\title{
Demagnetization cooling of a gas
}

\author{
M. Fattori* T. Koch, S. Goetz, A. Griesmaier, S. Hensler, J. Stuhler, and T. Pfau \\ E-mail:m.fattori@physik.uni-stuttgart.de Affiliation: 5. Physikalisches Institut, \\ Universität Stuttgart, Pfaffenwaldring 57, 70550 Stuttgart, Germany
}

\begin{abstract}
We demonstrate demagnetization cooling of a gas of ultracold ${ }^{52} \mathrm{Cr}$ atoms. Demagnetization is driven by inelastic dipolar collisions which couple the motional degrees of freedom to the spin degree. By that kinetic energy is converted into magnetic work with a consequent temperature reduction of the gas. Optical pumping is used to magnetize the system and drive continuous demagnetization cooling. Applying this technique, we can increase the phase space density of our sample by one order of magnitude, with nearly no atom loss. This method can be in principle extended to every dipolar system and could be used to achieve quantum degeneracy via optical means.
\end{abstract}

PACS numbers:

Keywords: 
Adiabatic demagnetization of a paramagnetic salt is the oldest method for reaching temperatures in solids significantly below $1 \mathrm{~K}$ [1]. Realized for the first time in the 30's [2], nowadays it is still used for its simplicity and flexibility. Based on the same principle of operation, nuclear demagnetization refrigerators make use of nuclear instead of electronic magnetic dipole moments [3]. Crucial for studies on magnetic phases in solids, they allow to cool the nuclear spin system well below $1 \mu \mathrm{K}$ [4, 5]. Also known as magnetocaloric effect [6], demagnetization cooling works in paramagnetic materials. They are constituted by particles with a total angular momentum quantum number $\mathrm{J}$ and with a permanent magnetic dipole $\mathrm{g} \mu(\mathrm{J}(\mathrm{J}+1))^{1 / 2}$ where $\mu$ is the unit of magnetic moment $\left(\mu_{\mathrm{B}}\right.$ for electrons and $\mu_{\mathrm{N}}$ for nuclei) and $\mathrm{g}>0$ is the spectroscopic splitting factor. If an external magnetic field is applied, such dipoles will try to minimize their energy aligning to it and generating a macroscopic magnetization $\mathrm{M}$ of the material. Quantum mechanically, this can be easily explained looking at the imbalance in the occupation probability $\propto \exp \left(-\mathrm{E}\left(\mathrm{m}_{\mathrm{J}}\right) / \mathrm{k}_{\mathrm{B}} \mathrm{T}\right)$ of every Zeeman state with energy $\mathrm{E}\left(\mathrm{m}_{\mathrm{J}}\right)=\mathrm{g} \mu \mathrm{Bm}_{\mathrm{J}}$ and with $\mathrm{m}_{\mathrm{J}}=-\mathrm{J},-\mathrm{J}+1, \ldots, \mathrm{J}-1$, J being the projection of the dipole moment along the magnetic field. For an intense magnetic field such that $\mathrm{g} \mu \mathrm{B} \gg \mathrm{k}_{\mathrm{B}} \mathrm{T}$ the probability of occupation of the state $\mathrm{m}_{\mathrm{J}}=-\mathrm{J}$ is nearly one and $\mathrm{M}$ saturates (see Fig. 1 a)). If after this isothermal magnetization the sample is isolated and $\mathrm{B}$ is reduced, the sample demagnetizes isoentropically and accordingly to the state equation $\mathrm{dQ}=\mathrm{TdS}=0=\mathrm{dE}+\mathrm{pdV}-\mathrm{BdM}$ we get $\mathrm{dE}=\mathrm{BdM}<0$ since volume variations are negligible in a solid [7]. In other words, the system has to do magnetic work to drive the demagnetization and consequently its internal energy and therefore its temperature decrease. This can be also understood from Fig. 1 b). When $\mathrm{k}_{\mathrm{B}} \mathrm{T} \sim \mathrm{g} \mu \mathrm{B}$, Zeeman states with $\mathrm{m}_{\mathrm{J}}>-\mathrm{J}$ can be occupied at the expense of the energy of the external degrees of freedom of the particles in the solid, with a net cooling effect. The cooling efficiency can be understood introducing the concept of the phonon reservoir, which includes the external degrees of freedom of the particles, and the spin reservoir to describe the internal state $\mathrm{m}_{\mathrm{J}}$. For high magnetic field, the spin degree of freedom is frozen and the spin reservoir specific heat $c_{\mathrm{S}}$ is negligible. The initial total energy $E_{i}$ is then equal to the phonon reservoir specific heat $c_{p}$ times the initial temperature $\mathrm{T}_{\mathrm{i}}$. When $\mathrm{k}_{\mathrm{B}} \mathrm{T} \sim \mathrm{g} \mu \mathrm{B}, \mathrm{c}_{\mathrm{s}}$ becomes of the order of $\mathrm{k}_{\mathrm{B}}$ and if a coupling between the two reservoirs exists, $E_{i}$ has to redistribute over a system with a total specific heat $c_{p}+c_{s}$. The final temperature is then $T_{\mathrm{f}}=\mathrm{T}_{\mathrm{i}}\left(\mathrm{c}_{\mathrm{p}} /\left(\mathrm{c}_{\mathrm{p}}+\mathrm{c}_{\mathrm{s}}\right)\right)$. Since in solids $\mathrm{c}_{\mathrm{p}} \ll \mathrm{c}_{\mathrm{s}}$, demagnetization can cool the sample by several orders of magnitude. 
a)

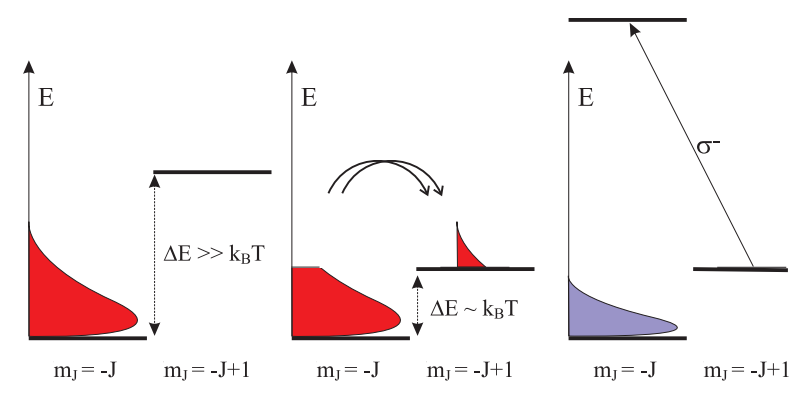

Figure 1: Description of demagnetization cooling. a) Initially the particles are polarized in the Zeeman state $\mathrm{m}_{\mathrm{J}}=-\mathrm{J}$ at high magnetic field $\mathrm{B}$. The mean energy $\mathrm{k}_{\mathrm{B}} \mathrm{T}$ is well below the Zeeman energy separation $\Delta \mathrm{E}=\mathrm{g} \mu_{\mathrm{B}} \mathrm{B}$. b) Reducing the external magnetic field until $\mathrm{k}_{\mathrm{B}} \mathrm{T} \approx \mathrm{g} \mu_{\mathrm{B}} \mathrm{B}$ allows demagnetization of the system. Part of the energy of the sample is transferred into Zeeman energy with a net cooling effect. c) In an atomic gas it is possible to use optical pumping to polarize the atoms back to $\mathrm{m}_{\mathrm{J}}=-\mathrm{J}$ leaving the temperature nearly unchanged. The system is ready for another cooling cycle.

Despite suggested by Kastler [8] 25 years before the first proposal on laser cooling [9], demagnetization processes so far have not been implemented in cooling techniques for gases. This is mainly due to the very weak coupling between the spin and the external degrees of freedom (phonon) reservoir in a system where the density is much smaller than in a solid. Very recently, our group has revisited this old idea and quantitatively analyzed its feasibility in the cooling of atoms that show large inelastic relaxation rates between magnetic substates 10]. During such relaxation, the sum of the quantum numbers $\mathrm{m}_{\mathrm{J} 1}$ and $\mathrm{m}_{\mathrm{J} 2}$ of two colliding atoms is not conserved and the demagnetization of the sample is allowed. Magnetic dipoledipole interaction can induce inelastic relaxations with a rate that drastically increase with the atomic magnetic moments. In particular the cross section for the inelastic single spin-flip (only one atom changes its $\mathrm{m}_{\mathrm{J}}$ value by one) is proportional to $\mathrm{J}^{3}$ 11]. In [10], particular attention has been paid to chromium atomic gases [12] because grounded $\mathrm{Cr}{ }^{7} \mathrm{~S}_{3}$ atoms possess very large magnetic dipole moments of six Bohr magnetons. This is due to the spin of six unpaired electrons in outer shells. As a consequence, in chromium the cross section for inelastic single spin flips is a factor of $\sim 200$ larger than in alkali atoms $(\mathrm{S}=\mathrm{J}=1 / 2)$.

In this Article, we demonstrate for the first time demagnetization cooling of a gas. ${ }^{52} \mathrm{Cr}$ atoms in the ${ }^{7} \mathrm{~S}_{3}$ ground state are initially polarized in the lowest energy Zeeman state 
$\left(\mathrm{J}=3, \mathrm{~m}_{\mathrm{J}}=-3\right)$ with a magnetic splitting $2 \mu_{\mathrm{B}} \mathrm{B}$ much larger than the temperature $\mathrm{T}$ of the sample. Reducing the magnetic field to values such that $3 / 2 \mathrm{k}_{\mathrm{B}} \mathrm{T} \approx 2 \mu_{\mathrm{B}} \mathrm{B}(100 \mu \mathrm{K} \sim 1$ Gauss $)$, transitions to higher energetic Zeeman substates, caused by dipolar relaxation collisions, cool the sample. In fact, the colliding atoms slow down because part of their kinetic energy is converted into Zeeman energy of the internal state. Single or double spin flips are possible. The main advantage of demagnetization cooling in a gas is that by using optical pumping it is possible to polarize the sample back to $\mathrm{m}_{\mathrm{J}}=-3$, constantly cooling the spin reservoir (see Fig. 1 c)).

Our atoms are stored in an optical dipole trap realized by a $1064 \mathrm{~nm}$ fiber laser. A horizontal $20 \mathrm{~W}$ beam focused to a $30 \mu \mathrm{m}$ waist generates a harmonic potential, independently of the magnetic substate, with $\omega_{y} / 2 \pi=\omega_{x} / 2 \pi=2 \mathrm{kHz}, \omega_{z} / 2 \pi=20 \mathrm{~Hz}$ and a depth of 200 $\mu \mathrm{K}$. Our experiments start with $10^{6}$ atoms polarized in $\mathrm{m}_{\mathrm{J}}=-3$. The details of the loading can be found in a previous work [12]. The initial temperature of the sample (temperatures are measured using absorption immaging and time of flight techniques) is $\sim 19 \mu \mathrm{K}$ due to plane evaporation and the external magnetic field is 1 Gauss. If we suddenly decrease the magnetic field to $50 \mathrm{mG}$ and let the system evolve, we observe a reduction of the temperature on a timescale of few seconds (see black squares in Fig. 2a)). After 5 seconds the equilibrium is achieved and we measure a final temperature of $16 \mu \mathrm{K}$. We have repeated the measurement keeping a 1 Gauss external magnetic field (see red circle in Fig. 2a)). As expected no temperature reduction has been detected. Since the energy of atoms is much larger than $\hbar \omega_{\mathrm{i}}(\mathrm{i}=\mathrm{x}, \mathrm{y}, \mathrm{z})$ the gas is classical and the specific heat per particle of the external degrees of freedom reservoir is $3 \mathrm{k}_{\mathrm{B}}$. Considering that the spin reservoir contribution is of the order of $\mathrm{k}_{B}$ we can then explain why the single step demagnetization can reduce the temperature by a factor of $\sim 3 / 4$. Another proof of the demagnetization of the system comes from the atom number measurement. Our detection scheme makes use of $\sigma^{-}$light resonant with the ${ }^{7} \mathrm{~S}_{3} \mathrm{~m}_{\mathrm{J}}=-3-{ }^{7} \mathrm{P}_{4} \mathrm{~m}_{\mathrm{J}}^{\prime}=-4$ transition. Absorption signal depend on the atomic distribution over the Zeeman substates and is stronger for atoms polarized in $\mathrm{m}_{\mathrm{J}}=-3$ (for $\mathrm{m}_{\mathrm{J}} \neq-3$ we have a different Zeeman detuning and a different coupling strength $\mathrm{CG}^{2}$ to the $\left.\mathrm{m}_{\mathrm{J}}^{\prime}=m_{\mathrm{J}}-1\right)$. As we can see in Fig. 2b) demagnetization results in a reduction of the detected atom signal. To exclude atom loss, we repolarize the sample with optical pumping after 5 seconds and verify that the detection signal is as large as at the beginning. Again red circles in Fig. 2b) show that this cooling step does not cause any extra loss respect 

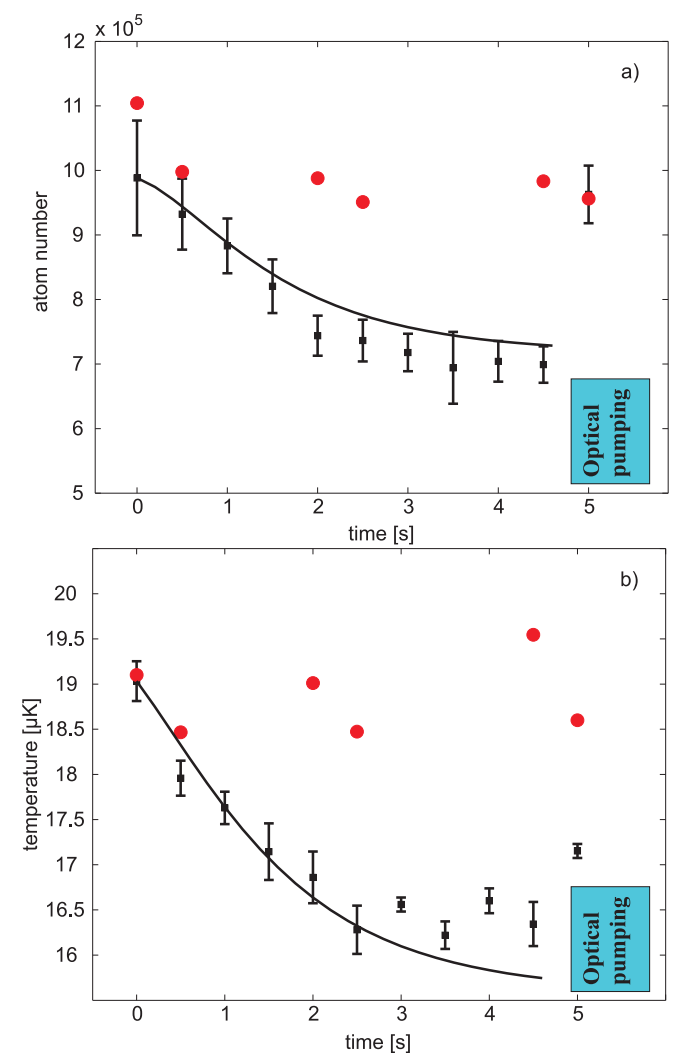

Figure 2: Single step demagnetization. At time zero the external magnetic field is suddenly switched from $1 \mathrm{G}$ to $50 \mathrm{mG}$. Temperature (in Fig. a)) and atom number (in Fig. b)) evolutions are represented by black squares. Every point is the result of three measurements average. Red circles show how the system evolves if the external magnetic field is kept at $1 \mathrm{G}$. Demagnetization results in temperature reduction and atomic depolarization, how our state selective measurement reveal. Switching on the optical pumping beam after 5 seconds slightly affects the temperature and pumps back all the atoms to the initial state. The black curve is the result of a theoretical calculation that takes into account demagnetization through all the seven magnetic sublevels. The input parameters are only the atom number, the initial temperature and the trap frequencies.

to the high magnetic field reference case. From the initial slope of the atom number and temperature curves in Fig. 2) it is possible to measure the depolarization rate at $\mathrm{t}=0$

$$
\frac{d N_{-3}}{d t}=-\beta_{\mathrm{dr}} \frac{N_{-3}^{2}}{\bar{V}}
$$

where $\bar{V}=\left(\sqrt{4 \pi \mathrm{k}_{\mathrm{B}} \mathrm{T} / m}\right)^{3} /\left(\omega_{x} \omega_{y} \omega_{z}\right)$ is the mean volume of the atomic cloud and $\beta_{\mathrm{dr}}=$ $\left\langle\left(\sigma_{1}+2 \sigma_{2}\right) v_{\text {rel }}\right\rangle_{\text {therm. }}$. Averaging over the inter-particle velocities we consider that the atomic depolarization can occur via single or double spin flip. The measurement of $\beta_{\mathrm{dr}} \sim 10^{-13} \mathrm{~cm}^{2} / \mathrm{s}$ 
results in good agreement with theoretical predictions based on $1^{\text {st }}$ order Born approximation for dipole-dipole interaction [11].

Performing optical pumping at the end of the demagnetization is crucial for preparing the system for another cooling step with smaller magnetic field and lower final temperature. This can be accomplished using $\sigma^{-}$polarized light on the ${ }^{7} \mathrm{~S}_{3}{ }^{7} \mathrm{P}_{3}$ optical dipole transition $(\lambda=427 \mathrm{~nm})$. In this way $\mathrm{m}_{\mathrm{J}}=-3$ is a dark state and its population is not affected by the pumping light. It is then possible to substitute several cooling steps with a continuous ramp of the magnetic field keeping on the optical pumping beam (OPB) during all the sequence. It has been proved theoretically that such strategy increases the cooling efficiency [10].

In order to preserve the polarization of the $\mathrm{OPB}\left(I_{\sigma^{+}} / I_{\sigma^{-}} \sim 1 / 1000\right)$ we have to keep the external magnetic field aligned to the OPB's propagation axis (y-axis). In fact, minimizing the heating effect of the OPB on the atoms we have been able to compensate residual magnetic fields $\mathrm{B}_{x}$ and $\mathrm{B}_{z}$ down to a few $\mathrm{mG}$.

The continuous cooling sequence starts with $10^{6}$ atoms at the temperature of $19 \mu \mathrm{K}$ with $\mathrm{B}_{y}=250 \mathrm{mG}$. The OPB is red detuned $40 \Gamma(\Gamma=2 \pi \times 5 \mathrm{MHz})$ from resonance and the total scattering rate is $\Gamma_{\mathrm{op}}=200$ photons/s. The best ramp of $\mathrm{B}_{y}$, optimized experimentally in order to maximize the final phase space density, results in a linear ramp down to $50 \mathrm{mG}$ in 7 seconds. Temperature and atom number evolution can be seen in Fig. 3). Red circles allow a comparison to the high magnetic field case. The final temperature is $11 \mu \mathrm{K}$. The atom loss is not related to the cooling mechanisms but most probably to finite background gas pressure. The cooling is insensitive to the detuning of the OPB both on the red and the blue side of the resonance in the 2-40 $\Gamma$ interval. Lower temperatures have not been achieved reducing $\mathrm{B}_{y}$ even further. In the next paragraph we analyze possible limitations.

As far as we are performing optical pumping largely detuned from resonance and the saturation parameter $\mathrm{s}$ is $\sim 10^{-5}$, the atom light scattering is mainly coherent [13]. Considering only the first order process, the light re-emitted by the atoms is blue shifted with respect to the pumping light by an amount $2 \mu_{\mathrm{B}} \mathrm{B}$. Due to the random direction of the emitted photon every cooling cycle causes an extra kick to the atoms with a total momentum $\hbar k$ where $k=2 \pi / \lambda$ is the light wavevector. However, simulations show that the ultimate temperature limit is slightly below the recoil temperature $\mathrm{T}_{\mathrm{rec}}=1 \mu \mathrm{K}[10]$. Reabsorption of the scattered photons could be also a serious limitation. The on-resonance light absorption cross section $\sigma=6 \pi / k^{2}$ holds the same even if the fluorescence photon is far from resonance with the 

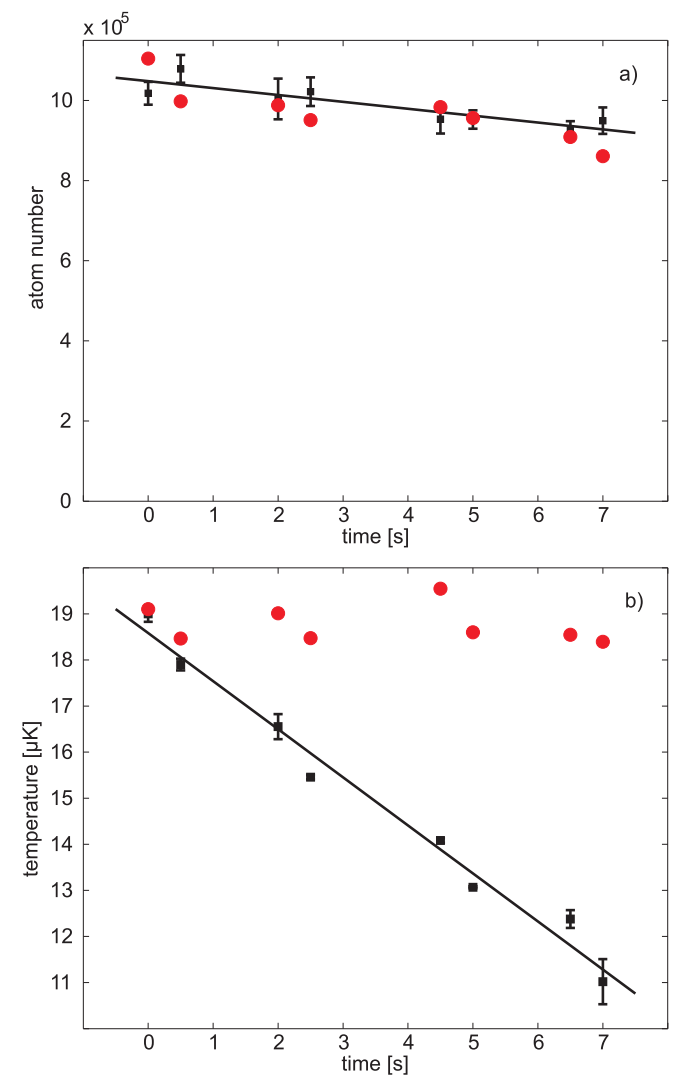

Figure 3: Continuous demagnetization cooling. The external magnetic field is linearly ramped from $250 \mathrm{mG}$ to $50 \mathrm{mG}$ in 7 seconds. Black squares shows atom number (Fig. a)) and temperature (Fig, b)) evolution. Every point is the result of 3 measurements. Red circles describe the evolution for a constant external magnetic field of $1 \mathrm{G}$. Note that the atom number reduction is not due to the cooling but due to single particle losses probably associated with finite lifetime in the dipole trap. Lines fit the cooling measurements.

bare atom: in fact the atoms are dressed by the optical pumping light and their fluorescence frequency matches the dressed states' energy difference [14]. Using the theoretical analysis presented in 15], which is valid for $\mathrm{kr} \geq 1$, where $\mathrm{r}$ is the interparticle separation, we can calculate the reabsorption probability. Note that the maximum density $\mathrm{n} \sim 10^{13}$ atoms $/ \mathrm{cm}^{3}$ at the end of the cooling and the optical pumping wavevector $\mathrm{k}$ fulfill the $\mathrm{kr} \geq 1$ condition. The reabsorption probability is

$$
p \sim \frac{6 \pi}{k^{2}} \frac{\Gamma_{\mathrm{op}}}{\omega_{\mathrm{D}}} \frac{1}{4 \pi\left\langle r^{2}\right\rangle} N
$$

The on-resonance cross section is reduced by a factor $\Gamma_{\mathrm{op}} / \omega_{\mathrm{D}} \sim 10^{-4}$ where $\omega_{\mathrm{D}} \sim 2 \times 10^{5}$ $\mathrm{s}^{-1}$ is the Doppler broadening at $10 \mu \mathrm{K} .\left\langle r^{2}\right\rangle$ is the mean square distance between two 
individual atoms in the trap $\left(\sim 3 \times 10^{-7} \mathrm{~m}^{2}\right)$ and $\mathrm{N} \sim 10^{6}$ the total number of trapped atoms. Consequently, for our experimental parameters the reabsorption probability is on the order of $p \sim 10^{-6}$. Theoretical analysis of the cooling including recoil energy, reabsorption, background gas and three body collisions predict a final temperature of $\sim 1 \mu \mathrm{K}[10]$. We therefore conclude that a heating mechanism is currently limiting us, most probably coming from a non perfect control of the fields $\mathrm{B}_{z}$ and $\mathrm{B}_{x}$. We observe in fact a drift in the currents that optimize such transversal fields. Several checks are necessary during one day measurement. This could be due to instability of our current generators or to random magnetization of our steel chamber during the switching of the magnetic trap we use to prepare our atomic samples. External ac fields of the order of few $\mathrm{mG}$ could also be limiting us.

The development of such cooling technique helps us on our route to the chromium BEC. Our measurements show that the efficiency of demagnetization cooling $\chi=$ $-\ln \left(\rho_{\mathrm{f}} / \rho_{\mathrm{i}}\right) / \ln \left(\mathrm{N}_{\mathrm{f}} / \mathrm{N}_{\mathrm{i}}\right)$ associated to the gain in phase space density over the loss in atom number is $\sim 11$. So far, this is much better than the optimum value achieved $(\sim 4)$ using evaporative cooling. By this our starting conditions in the optical dipole trap result better, with nearly one order of magnitude higher phase space density at the same atom number.

In the atom optics community, demagnetization cooling belongs to the family of laser cooling techniques in external fields [16, 17]. What is new is the mechanism that performs the selection of higher energy atoms. In our case, we make use of dipole-dipole inelastic collisions, while in other experiments this is performed via optical Raman transitions [18, 19]. Such a technique, applied to Cs and performed in a far-off-resonant lattice has lead to final phase space density of 1/30 [20]. Higher values are prevented by inelastic hyperfine changing collisions occurring between two atoms at the same lattice site. Cr has no hyperfine structure and this will not be the limiting effect in the challenge of achieving Bose Einstein condensation via all-optical means with demagnetization cooling. As shown in 21] crucial for the suppression of reabsorption will be working in the festina lente regime, where reabsorption is prevented if the optical pumping rate $\Gamma_{\text {op }}$ is smaller than the trapping frequency. Such regime has been theoretically proved to work if $\mathrm{kr} \geq 1$ [15] ( for higher densities radiative interatomic collisions start to play an important role 22]). Considering our optical pumping light wavelength and the mass of $\mathrm{Cr}$ we deduce that such condition is fulfilled for typical condensation temperatures below $3 \mu \mathrm{K}$. All optical BEC with demagnetization cooling is 
then in principle possible with a more accurate control of the external magnetic fields.

Demagnetization or the general depolarization cooling is an important tool that can in principle be applied to other bosonic and fermionic systems. Promising is its use in the cooling of heteronuclear molecules with a permanent electric dipole moment. Note that as dipole-dipole interaction is long range, in the partial waves decomposition of the cross

section all the orders contribute, even in the limit of zero collision energy. Therefore, also fermionic dipolar molecules and fermionic atoms can demagnetize and thermalize via elastic dipole-dipole interaction.

\section{Acknowledgments}

We thank our atom optics group for encouragement and practical help. This work was supported by the Alexander von Humboldt Foundation and the German Science Foundation (DFG) (SPP1116 and SFB/TR 21).

\section{Competing financial interests}

The authors declare that they have no competing financial interests.

* Electronic address: m.fattori@physik.uni-stuttgart.de

[1] Lounasmaa, O. V. Experimental Principles and Methods Below $1 \mathrm{~K}$ (Academic Press, 1974).

[2] De Haas, W. J. \& Kramers, H. A., Physica 1, 1 (1933).

[3] Kurti, F. S. N., Robinson, F. N. \& Spohr, D. A. Nature 178, 450 (1956).

[4] Oja, A. S. \& Lounasmaa, O. V. Rev. Mod. Phys. 69, 1 (1997).

[5] Touriniemi, J. T. \& Knuuttila, T. A. Physica B 280, 474 (2000).

[6] Pecharsky, V. K. \& Gschneidner, K. A. Phys. Rev. Lett. 78, 4494 (1997).

[7] Morrish, A. H. The Physical Principles of Magnetism (John Wiley and Sons, 1983).

[8] Kastler, A. Le Journal de Physique et le Radium 11, 255 (1950).

[9] Hänsch, T. W. \& Schawlow, A. Opt. Comm. 13, 68 (1975).

[10] Hensler, S., Greiner, A., Stuhler, J., \& Pfau, T. Europhys. Lett. 71, 918 (2005).

[11] Hensler, S. et al. Appl. Phys. B 77, 765 (2003).

[12] Griesmaier, A., Werner, J., Hensler, S., Stuhler, J., \& Pfau, T., Phys. Rev. Lett. 94, 160401 (2005).

[13] Cohen-Tannoudji, C., Dupont-Roc, J., \& Grynberg, G. Atom-photon interactions: Basic Pro- 
cesses and Applications (John Wiley \& Sons, 1992), 1st ed., pp. 382-383.

[14] Olshan'ii, Y. C. M. \& Dalibard, J. Proceedings of the 12th International Conference on Laser Spectroscopy (World Scientific, Singapore, 1996).

[15] Castin, Y. \& Lewenstein, M. Phys. Rev. Lett. 80, 5305 (1998).

[16] Cirac, J. I. \& Lewenstein, M. Phys. Rev. A 52 (1995).

[17] Cirac, J. I., Lewenstein, M., \& Zoller, P. Europhys. Lett. 35 (1996).

[18] Lee, H. J., Adams, C. S., \& Chu, S. Phys. Rev. Lett. 76, 2658 (1996).

[19] Kerman, A. J., Vuletic, V., Chin, C., \& Chu,. S Phys. Rev. Lett. 84, 439 (2000).

[20] Han, D.-J. et al. Phys. Rev. Lett. 85, 724 (2000).

[21] Wolf, S., Oliver, S. J., \& Weiss, D. S. Phys. Rev. Lett. 85, 4249 (2000).

[22] Hijmans, T. W. \& Burin, A. L Phys. Rev. A 54, 4332 (1996). 